Strahlenther Onkol 2013 · 189:339-339

DOI 10.1007/s00066-013-0319-0

Online publiziert: 20. Februar 2013

(c) Springer-Verlag Berlin Heidelberg 2013

\author{
Th. Herrmann ${ }^{1} \cdot$ R. Sauer ${ }^{2}$ \\ ${ }^{1}$ Dresden \\ ${ }^{2}$ Universitätsklinikum Erlangen
}

\title{
Professor Árpád Mayer zum 70. Geburtstag
}

lenbiologischer Art am Petrow-Institut in Leningrad absolviert. Nach der Facharztanerkennung für Radioonkologie in Ungarn bildete er sich 1975/76 in Zürich in der MDR-Brachytherapie und der CTgestützten Bestrahlungsplanung unter der Leitung von Professor Horst weiter. 1984 erhielt er den Grad eines PhD in Budapest verliehen und wurde 1993 zum Chefarzt der radioonkologischen Abteilung am Uzsoki-Hospital berufen. Seit 1994 gehört er als Associate Professor zum medizinischen Lehrkörper an der Semmelweis-Universität in Budapest.

Das spezifische Interesse von Árpád Mayer galt immer der Brachytherapie in ihren verschiedenen Techniken und biologischen Wirkungen. So hat er sowohl im Bereich der LDR-, als auch der selten angewandten MDR-Brachytherapie bedeutende wissenschaftliche Arbeit geleistet und verbesserte damit besonders in den letzten Jahren die Anwendung der HDRBrachytherapie in Ungarn wesentlich.

Professor Mayer hat neben vielen Auszeichnungen durch nationale und internationale Gesellschaften eine Fülle von verantwortungsvollen Aufgaben in der ungarischen Radioonkologie und in anderen wissenschaftlichen Gremien wahrgenommen. Unter anderem war er jahrelang Generalsekretär der ungarischen Krebsgesellschaft, dann auch ihr Präsident.

Der deutschen Radioonkologie ist Árpád Mayer über viele Jahrzehnte eng verbunden. Nicht nur, dass er 1995 das erste ungarische DEGRO-Mitglied war, sondern es verbinden ihn enge kollegiale, ja freundschaftliche Beziehungen zu den großen deutschen Kliniken in Leipzig, Dresden, Essen, Tübingen, München und Erlangen. Er hat in seinem Be- rufsleben stets Sorge dafür getragen, dass die Verbindungen zwischen Ungarn und Deutschland - anfangs zu den beiden deutschen Staaten, später zu Gesamtdeutschland - immer enger wurden. 1996 wurde er auch Mitherausgeber unserer Zeitschrift Strahlentherapie und Onkologie.

Diese Würdigung wäre nicht vollständig, wenn hier nicht das besondere Interesse Árpád Mayers an der deutschen Kultur erwähnt würde, besonders seine Liebe zur Musik Richard Wagners. Dies festigte zusätzlich seine Verbindungen zu Deutschland. Regelmäßig ist er deshalb bei den Bayreuther Festspielen anzutreffen und das wird auch im Wagner-Jahr 2013 der Fall sein.

Wir danken Árpád Mayer auch im Namen der DEGRO für seine immer währenden Bemühungen, zwischen der ungarischen und der deutschen Radioonkologie Brücken zu schlagen und hoffen auch in der Zukunft auf viele gute Begegnungen mit ihm, und zwar nicht nur auf dem "Grünen Hügel“, sondern als aktiver Teilnehmer auf den DEGRO-Kongressen.

Thomas Herrmann, Dresden

Rolf Sauer, Erlangen

\section{Korrespondenzadresse}

Prof. Dr. Th. Herrmann

Elbeweg 8, 01326 Dresden

th.Herrmann@macbay.de

Prof. Dr. R. Sauer

Universitätsklinikum Erlangen

Universitätsstr. 27, 91054 Erlangen

rolf.sauer@uk-erlangen.de traler Arbeitsplatz. Vorher hatte er Ausbildungsphasen biophysikalischer Art an der Karls-Universität in Prag und strah- 